\title{
Overriding auditory attentional capture
}

\author{
Polly Dalton \\ Royal Holloway University of London, Egham, England \\ AND \\ NiLli LAVIE \\ University College London, London, England
}

\begin{abstract}
Attentional capture by color singletons during shape search can be eliminated when the target is not a feature singleton (Bacon \& Egeth, 1994). This suggests that a "singleton detection" search strategy must be adopted for attentional capture to occur. Here we find similar effects on auditory attentional capture. Irrelevant highintensity singletons interfered with an auditory search task when the target itself was also a feature singleton. However, singleton interference was eliminated when the target was not a singleton (i.e., when nontargets were made heterogeneous, or when more than one target sound was presented). These results suggest that auditory attentional capture depends on the observer's attentional set, as does visual attentional capture. The suggestion that hearing might act as an early warning system that would always be tuned to unexpected unique stimuli must therefore be modified to accommodate these strategy-dependent capture effects.
\end{abstract}

Previous research has shown that visual search can be interrupted by the presence of an irrelevant feature singleton, such as a unique red stimulus among green stimuli (Pashler, 1988; Theeuwes, 1991, 1992). We have recently established a similar phenomenon in the auditory domain, demonstrating that irrelevant auditory singletons (i.e., sounds that are unique on a particular auditory dimension such as frequency or intensity) tend to capture attention during auditory search for targets defined on a different dimension such as duration (Dalton \& Lavie, 2004). This effect generalized across four different types of auditory singletons (high frequency, low frequency, high intensity, and low intensity), three different target dimensions (intensity, frequency, and duration) and two different auditory search tasks (requiring either target detection or target discrimination). The effects therefore seem to reflect attentional capture (AC) by unique items in general. We also found that, in a way similar to visual $\mathrm{AC}$, auditory AC can cause interference or facilitation, depending on whether the irrelevant singleton feature is presented within a distractor or target item.

The fact that nontarget singletons can interfere with search despite being defined on a task-irrelevant dimension may at first appear to suggest that AC is an involuntary stimulus-driven process. However, evidence suggests that visual $\mathrm{AC}$ in fact depends on the attentional allocation strategy adopted by the observer during search. For example, Folk, Remington, and Johnston (1992) found that singleton cues that were unique on the dimension of abrupt onset captured spatial attention only if the target of the subsequent search task was also defined by abrupt onset. When the target was defined on the dimension of color, the invalid abrupt-onset singleton did not capture attention. Similarly, singleton cues defined by color captured attention if the subsequent target was also defined by color (even if it was of a different color from the singletons) but not if it was defined by onset. It therefore seems that singleton precues presented among other competing items will only capture spatial attention if the participant's attentional set includes the dimension on which the singleton is defined (see also Folk \& Remington, 1998; Folk, Remington, \& Wright, 1994).

The above studies presented the singleton in a precue display. However, there is also evidence that $\mathrm{AC}$ by singletons presented as part of a visual search array depends on subjects adopting a singleton detection strategy in their search for the target, in which they allocate attention toward any unique items in the search display rather than focus their search on the target feature (a strategy known as feature search mode). In most of the tasks used in the visual search AC paradigm, this is an efficient search strategy, as the target itself is a feature singleton and will thus be identified by the "odd one out" search. However, a singleton detection strategy also leaves the search open to AC by an irrelevant singleton distractor. By contrast, if participants are prevented from using a singleton detection strategy, visual AC is eliminated. For example, Bacon and Egeth (1994) showed that the interference due to color singletons found in Theeuwes (1992) was eliminated when the target was not always a singleton, either because there was more than one target item in the array (Experiment 2) or because nontargets of several different forms appeared

P. Dalton, polly.dalton@rhul.ac.uk 
in each array (Experiment 3). Both of these experiments showed significantly reduced interference by an irrelevant color singleton, compared with an experiment in which the target was a reliable form singleton (Experiment 1). Moreover, Experiments 2 and 3 both included a subset of trials in which there was only one unique target present in the display (as in Bacon \& Egeth's Experiment 1 and Theeuwes, 1992). However, even an analysis of these trials alone found no significant singleton interference, presumably because the singleton-target trials were intermixed at random with trials without singleton targets, encouraging participants to use a feature search strategy throughout the experiment. Thus, it seemed that participants must be using a singleton detection strategy in order for visual AC by irrelevant singletons to take place.

The present experiments examine whether auditory $\mathrm{AC}$ also depends on the search strategy adopted by the listener. Specifically, they are designed to test whether interference effects due to auditory AC can be eliminated when subjects are forced into a feature search strategy, rather than a singleton detection one. As well as providing a comparison between the visual and auditory versions of the $\mathrm{AC}$ effect, this study has interesting implications for theories of control of auditory attention. Because hearing can scan the environment in all directions while other senses are focused on more restricted areas of space, it is often argued that hearing might have evolved, at least in part, to act as an early warning system, alert to unexpected events (e.g., Scharf, 1998). If so, we might expect hearing to be more open to AC than is vision, in which case auditory AC might be less strategy-dependent than visual AC, in the sense that it could occur even when subjects were not using a singleton detection strategy.

In order to assess the effects of search strategy on auditory AC, we modified Dalton and Lavie's (2004) paradigm to allow us to manipulate whether or not a singleton detection strategy was available to the participant. To ensure that the manipulation of search strategy had an effect solely on search processes, without altering the response demands of the task, we asked participants to search for targets defined on one dimension (frequency) and make discrimination responses on a different dimension (duration). This modified task provides an auditory analogue of the task used by Theeuwes (1992), the basis of Bacon and Egeth's (1994) task, in which participants searched for a target defined by shape and discriminated between different orientations of lines contained within the shape. Irrelevant singletons in the present tasks were defined as having a higher intensity than the other sounds. ${ }^{1}$ We expected that these high-intensity singletons would produce a relatively strong capture effect, so that any modulation of this effect by manipulations of attentional allocation strategies would not be attributable to the irrelevant singletons simply being weak competitors for attention.

As in our previous experiments (Dalton \& Lavie, 2004), we used sequentially presented auditory search arrays. In both studies this was motivated primarily by consideration of the ways in which auditory information is processed. Research now agrees that, unlike the visual system, the auditory system processes spatial location with lower priority than other stimulus attributes, such as timing and frequency (e.g., Kubovy, 1981). In line with this observation, there is evidence that auditory information tends to dominate visual information in tasks involving temporal judgments, whereas visual information tends to dominate in tasks involving spatial judgments (e.g., Welch, DuttonHurt, \& Warren, 1986). Some researchers therefore propose that temporal separation of auditory stimuli might in some ways be comparable with spatial separation of visual stimuli (e.g., Kubovy, 1981). In line with this view, most previous research into focused auditory attention and the effects of auditory distractors has used temporal rather than spatial search arrays (e.g., Cusack \& Carlyon, 2003; Dyson \& Quinlan, 2003; Woods, Alain, Diaz, Rhodes, \& Ogawa, 2001).

Sequential search arrays have also been used in visual experiments, typically to examine the effects of attentional allocation to targets - for example, in the attentional blink paradigm (Broadbent \& Broadbent, 1987; Raymond, Shapiro, \& Arnell, 1992) — but also more recently to examine visual AC by to-be-ignored singletons. For example, there is evidence from studies using the attentional blink paradigm that a to-be-ignored singleton (i.e., an ignored first target or a colored box around a nontarget letter) can interfere with recall of the second target, suggesting that attention was allocated to the supposedly ignored singleton item (e.g., Chun, 1997; Folk, Leber, \& Egeth, 2001; Maki \& Mebane, 2006; Wee \& Chua, 2004). However, reliable effects in these studies were only produced by items that were defined on the same dimension as the targets (e.g., they were both color singletons; see also Ghorashi, Zuvic, Visser, \& Di Lollo, 2003) and it is therefore unclear whether the results reflect AC by the singletons or deliberate attentional allocation toward these singletons because they possess a task-relevant feature. By contrast, Dalton and Lavie (2006) showed AC during rapid serial visual presentation (RSVP) size discrimination task by singletons defined on the task-irrelevant dimension of color, suggesting that visual $\mathrm{AC}$ can be found in sequential search arrays. However, it remains possible that Dalton and Lavie's (2006) findings of temporal visual AC also depend on the subjects adopting a singleton detection strategy. This issue is currently under investigation in our lab.

Experiment 1 establishes singleton interference within our modified auditory search task, replicating the findings of Dalton and Lavie (2004). The subsequent experiments then ask whether it is possible to eliminate this interference by adding another type of nontarget to the search arrays (Experiment 2), or by adding more than one target to the arrays (Experiment 3). As was the case in Bacon and Egeth's (1994) study, both of these manipulations will prevent the target from being a reliable frequency singleton, forcing subjects to adopt a feature search strategy, rather than a singleton detection one. The critical question is whether the interference effects of irrelevant auditory singletons will be eliminated in such situations. Finally, Experiment 4 investigates possible effects of stimulus variation on singleton interference effects. 


\section{EXPERIMENT 1}

This experiment aimed to replicate our previous findings of singleton interference using a version of Dalton and Lavie's (2004) auditory search task that was modified so that the response-defining dimension (duration) was different from the target-defining dimension (frequency). Participants searched sequences of five sounds, presented one after another, for a target that was present on each trial. The target was defined as having a lower frequency than the nontargets, and participants responded at the end of each sequence according to whether this low frequency target was of long or short duration. An irrelevant singleton sound, defined as having a higher intensity than the other sounds, was present on half of the trials. The nontargets were all of the same frequency, providing a homogeneous background and making the target a reliable frequency singleton. This should have allowed participants to search the array using singleton detection mode, leaving them open to interference by the irrelevant singleton.

\section{Method \\ Participants. Participants in all the experiments reported here were students at University College London and were paid $£ 5$ for participation. All were under 35 years old and reported normal hearing. Twelve participants took part in Experiment 1. Two were replaced due to near-chance level performance ( $41 \%$ and $50 \%$ error rates); a third was replaced due to a mean reaction time (RT; $580 \mathrm{msec}$ ) that was over three standard deviations $(S D \mathrm{~s})$ from the group mean (group $M=310 \mathrm{msec}, S D=48.7 \mathrm{msec}$ ). \\ Apparatus and Stimuli. The experiments were created and run on a PC using E-Prime (Version 1.0, Psychology Software Tools, Inc., Pittsburgh, PA). Auditory stimuli were created using SoundEdit 16 (Macromedia, Inc., San Francisco, CA) and presented through open- cup headphones (beyerdynamic, Heilbronn, Germany). \\ Targets were defined by frequency being lower $(480 \mathrm{~Hz})$ than nontargets $(780 \mathrm{~Hz})$. Singletons were presented at the nontarget fre- quency $(780 \mathrm{~Hz})$ and were of higher intensity - approximately $85 \mathrm{~dB}$ sound pressure level (SPL) - than targets and nontargets (approxi- mately $73 \mathrm{~dB}$ SPL). Intensities were measured using an artificial ear and SPL meter (Brüel \& Kjær) at the participants' ears. Because the task was to respond according to the duration of the target, all sounds could have durations of either $100 \mathrm{msec}$ or $300 \mathrm{msec}$. Sounds were separated by different interstimulus intervals (ISIs) depending on their durations. The total duration of stimulus presentation and ISI (i.e., stimulus onset asynchrony, or SOA) was kept constant at $400 \mathrm{msec}$ : Sounds of $100 \mathrm{msec}$ were followed by 300 -msec ISIs, and sounds of $300 \mathrm{msec}$ were followed by 100 -msec ISIs. Frequencies, intensities, and durations were chosen to be easily discriminable, as verified by pilot testing.}

Design and Procedure. The start of each trial was signaled by the display of the word Ready on a screen for $500 \mathrm{msec}$. This was followed by a sequence of five successive sounds. ${ }^{2}$ Participants searched each short sequence for a target sound of lower frequency than the nontargets and responded according to whether the tone was of long or short duration. A target appeared on every trial in either the third or fourth position with equal probability, and was just as likely to be long in duration as short. Nontarget durations were assigned as short or long at random. A high-intensity singleton appeared on $50 \%$ of trials, either directly before or directly after the target, and with long or short duration (both with equal probability).

Response timing began at the offset of the last tone of the sequence, which was signaled by the appearance of a question mark at the center of the screen. Participants responded according to the duration of the target sound, pressing 1 for long target or 2 for short target, using the right index and middle fingers, respectively, on the number keypad. Visual feedback was provided at the end of each trial, either after a response had been collected, or after 3,000 msec, if no response had been detected. The feedback screen displayed either the word Correct presented in blue, the word Incorrect presented in red, or the phrase No response detected in red. This screen display lasted 1,500 msec, after which time the Ready display was presented in preparation for the next trial. Participants were instructed to focus on the target frequency dimension and to ignore any sounds of the irrelevant frequency. They were informed that there might be some odd sounds presented at the irrelevant frequency and were warned that their performance might be harmed if they failed to ignore the irrelevant distractors.

A 64-trial block included a fully counterbalanced mix of the following factors and their combinations: target position (3rd or 4th in sequence), target duration (long or short), singleton duration (long or short), and singleton condition (absent vs. before-target vs. aftertarget). Six experimental blocks were run, preceded by two practice blocks, each containing 16 trials. In the first practice block, there was no time limit for responses and there was also a break between each trial to allow the experimenter to provide more detailed feedback if necessary. The second practice block followed exactly the same procedure as the experimental blocks.

\section{Results and Discussion}

RTs. For the RT analysis, incorrect responses were excluded, as were RTs longer than 1,500 msec. These criteria were used in all the experiments reported in this paper. Table 1 shows mean RTs and error rates as a function of singleton condition (absent vs. before-target vs. after-target) for Experiments 1 and 2. A one-way withinsubjects ANOVA on the RT data with the factor of singleton condition (absent vs. before-target vs. after-target) revealed a significant main effect $\left[F(2,22)=5.84, M S_{\mathrm{e}}=\right.$ $250.02, p<.01] .^{3} F$ contrasts confirmed that, by comparison with the singleton-absent condition, RTs were significantly slower both when the singleton occurred before the target $\left[F(1,11)=10.28, M S_{\mathrm{e}}=447.43, p<.01\right]$ and when it occurred after the target $\left[F(1,11)=6.01, M S_{\mathrm{e}}=\right.$ $688.94, p<.05]$ (see Table 1 ). There was no difference in RTs between singletons appearing before the target $(M=$ $320 \mathrm{msec})$ and after the target $(M=319 \mathrm{msec}, F<1)$. These findings are in line with the singleton interference demonstrated in our previous experiments (Dalton \& Lavie, 2004) and suggest that the high-intensity singleton captured attention during the search for the low frequency target, despite being irrelevant to that search.

In the present experiment, as in previous studies of AC in RSVP tasks (e.g., Chun, 1997; Dalton \& Lavie, 2004, 2006; Folk, Leber, \& Egeth, 2001, 2002; Maki \& Mebane, 2006; Wee \& Chua, 2004), we collected responses at the end of the stimulus sequences rather than allowing participants to

Table 1

Averages of Subjects' Mean Reaction Times (RTs, in Milliseconds) With Standard Errors $(S E)$ and Error Rates (\%E) for Experiments 1 and 2, As a Function of Singleton Condition Singleton Condition

\begin{tabular}{|c|c|c|c|c|c|c|c|c|c|}
\hline & \multicolumn{9}{|c|}{ Singleton Condition } \\
\hline & \multicolumn{3}{|c|}{ Absent } & \multicolumn{3}{|c|}{ Before Target } & \multicolumn{3}{|c|}{ After Target } \\
\hline & RT & $S E$ & $\% \mathrm{E}$ & RT & $S E$ & $\% \mathrm{E}$ & RT & $S E$ & $\% \mathrm{E}$ \\
\hline Experiment 1 & 301 & 13 & 5 & 320 & 15 & 6 & 319 & 16 & 5 \\
\hline Experiment 2 & 368 & 30 & 12 & 367 & 31 & 14 & 363 & 27 & 13 \\
\hline
\end{tabular}


respond during stimulus presentation. However, the average RTs found in the present experiment $(M=310 \mathrm{msec})$ and in our previous experiments (Dalton \& Lavie, 2004) are relatively fast in terms of choice RTs (e.g., Laming, 1968). Thus the capture effects in the present experiment are unlikely to be related to memory/retrieval processes, because if subjects were retrieving the whole sequence from memory at the end of each trial we would expect the RTs to be longer than the average for straightforward choice RTs. By contrast, the short RTs observed here are more likely to indicate search RTs, and the singleton effect is thus likely to reflect interference in this search, rather than interference in memory/retrieval. This issue is addressed further in Experiment 4, which found AC effects using a task in which RTs were measured from target presentation.

The idea that the interference seen here is due to online $\mathrm{AC}$ has a precedent in studies of auditory attentional blink, in which responses were also collected at the end of the sequence. For example, Duncan, Martens, and Ward (1997) demonstrated that, similar to visual attention, auditory attention can be subject to an attentional blink, such that once attention has been allocated to one item there is a period of time during which it cannot be reallocated to subsequent items (see also Arnell \& Larson, 2002; SotoFaraco \& Spence, 2002). Thus, online attentional allocation to one item can interfere with processing of another item in the same sequence - a finding that appears similar to the interference in online attending seen in the present experiment. We note, however, that the attentional blink research assesses the consequences of attending deliberately to target stimuli, and so does not provide information about capture of attention by irrelevant stimuli. In addition, since typically participants are asked to respond to the first item as well as to direct their attention to it, the attentional blink it causes is likely to involve both response-related (and possibly memory-related) effects (e.g., Jolicœur, 1998). By contrast, the present demonstrations of auditory AC show interference effects associated with the presence of irrelevant items that do not require responses. These effects can thus only be explained in terms of attentional allocation processes.

Errors. There were few errors $(M=5 \%)$ showing few variations between singleton conditions (absent vs. beforetarget vs. after-target, $F<1$; see Table 1 ).

In conclusion, the present experiment shows significant $\mathrm{RT}$ interference associated with the presence of irrelevant singleton distractors. This replicates our previous findings of AC by auditory singletons (Dalton \& Lavie, 2004), using a slightly modified version of the task in which subjects' responses are based on a nondefining attribute.

The finding here (and in Dalton \& Lavie, 2004) of equivalent effects due to singletons appearing both before and after the target provides a contrast with previous studies of visual AC in RSVP tasks, which typically find effects of singletons occurring before but not after the target (e.g., Dalton \& Lavie, 2006; Folk et al., 2002). We note, however, that, in line with visual AC studies, some of our previous experiments have shown that the presence of a singleton before the target can be more detrimental to accuracy than the presence of a singleton after the target
(Dalton \& Lavie, 2004, Experiments 3B, 4A, and 4B). It seems likely that these effects were not replicated here because of the low error rates in the present experiment.

\section{EXPERIMENT 2}

In Experiment 2, we asked whether the singleton interference effect found in Experiment 1 was due to participants adopting a singleton detection strategy in their search for the target. Because the targets in Experiment 1 were reliable frequency singletons, a singleton detection strategy would have been effective for identifying potential targets, but would also have left participants open to interference by the irrelevant distractor singleton. In Experiment 2, participants searched for a target that was present on each trial (with the same frequency as the targets in Experiment 1) and once again responded according to target duration. However, in an important change from Experiment 1 , the nontargets now included sounds of lower frequency than the target, in addition to the sounds of higher frequency than the target used before. This should have prevented participants from using a singleton detection strategy, as the target was no longer a reliable frequency singleton. Instead, participants were encouraged to adopt a feature search mode, in which they would focus on the particular specified target frequency rather than simply scanning the array for singletons on any dimension. If, like visual AC, auditory AC depends on singleton detection strategy, then the auditory intensity singleton should no longer have captured attention in this task.

\section{Method}

Participants. Twelve new participants took part in the experiment. Four were replaced due to near-chance level performance $(50 \%, 50 \%, 49 \%$, and $44 \%$ error rates).

Stimuli and Apparatus. The stimuli used were similar to those used in Experiment 1, except that nontargets and singletons were now of either high frequency $(780 \mathrm{~Hz})$ or low frequency $(180 \mathrm{~Hz})$. (Recall that nontargets and singletons in Experiment 1 had all been of high frequency.) The apparatus was identical to that used in Experiment 1.

Design and Procedure. The design was similar to that of Experiment 1 , except that nontargets and singletons could be of either high or low frequency. Frequencies were assigned at random with the following constraints: Each sequence contained at least one stimulus of high frequency and one of low frequency, as well as the medium frequency target. The high-intensity singletons were just as likely to have high frequencies as they were to have low frequencies, and both types of singleton appeared before and after the target with equal probability. Possible target positions (third or fourth in the sequence) were identical to those in Experiment 1. The procedure was identical to that of Experiment 1, except that the experimental trials were run in four blocks of 96 for reasons of counterbalancing.

\section{Results and Discussion}

RTs. A one-way within-subjects ANOVA on the RT data with the factor of singleton condition (absent vs. beforetarget vs. after-target) revealed no significant main effect ( $F<1$ for both comparisons; see Table 1). This finding stands in contrast to the results of Experiment 1, in which singletons were found to cause reliable interference effects. In order to compare the singleton effects between Experiments 1 and 2, we ran a two-way mixed-model 
ANOVA using the within-subjects factor of singleton presence (present vs. absent) and the between-subjects factor of experiment (1 vs. 2). This analysis confirmed that the singleton effect in Experiment $1(M$ effect $=19 \mathrm{msec})$, in which participants were able to use a singleton detection strategy, was significantly reduced in Experiment 2 ( $M$ effect $=-3 \mathrm{msec}$ ), in which this strategy was prevented $\left[F(1,22)=5.36, M S_{\mathrm{e}}=264.53, p<.05\right]$. There was also a numerical trend for slower RTs in Experiment 2 $(M=366 \mathrm{msec})$ than in Experiment $1(M=310 \mathrm{msec})$, although this was not significant $\left[F(1,22)=3.05, M S_{\mathrm{e}}=\right.$ $12,452.86, p=.095]$.

Errors. Error rates in this experiment $(M=13 \%)$ were higher overall than those in Experiment $1(M=5 \%)$ $\left[F(1,22)=7.56, M S_{\mathrm{e}}=94.29, p<.05\right]$. Nevertheless, as in Experiment 1, there was little variation in errors between singleton conditions (absent vs. before-target vs. after-target) $\left[F(2,22)=2.64, M S_{\mathrm{e}}=5.72, p=.094\right.$; see Table 1].

Although the higher number of errors in Experiment 2 than in Experiment 1 might suggest that Experiment 2 was more difficult, the numerical trend for slower RTs in Experiment 2 was not statistically significant. Because this is primarily an RT experiment, in which assessment of singleton interference is based on RT performance, the effect of task difficulty on RTs is more relevant than possible effects of task difficulty on error rates. Nevertheless, because there was a numerical trend for slower RTs in Experiment 2 than in Experiment 1, it is possible that Experiment 2 might not have been sufficiently sensitive to reveal RT effects of singleton interference. Reanalysis of Experiment 1 casts some doubt on this possibility: Despite a trend for worse performance in the first half of the experiment $(M=347 \mathrm{msec})$ compared with the second $(M=318 \mathrm{msec})$, there was no evidence for a difference in the level of singleton interference between the first ( $M$ interference effect $=16 \mathrm{msec})$ and second $(M$ interference effect $=18 \mathrm{msec}$ ) halves. Moreover, reanalysis of the capture effects by a high-intensity singleton similar to that used here in a previous experiment (Dalton \& Lavie, 2004, Experiment 1A) also showed equivalent capture effects in the first $(M$ effect $=28 \mathrm{msec})$ and second $(M$ effect $=$ $31 \mathrm{msec}$ ) halves, despite significantly worse performance in the first half $(M=279 \mathrm{msec})$ than in the second half $(M=249)\left[F(1,7)=13.65, M S_{\mathrm{e}}=778.27, p<.01\right]$. This issue is examined more directly in Experiment 3.

\section{EXPERIMENT 3}

Experiment 3 was run in order to examine the effects of participants' search strategies in a design that does not lead to any increase in task difficulty. The majority $(70 \%)$ of trials in Experiment 3 contained search arrays in which there were two target sounds - in contrast to Experiment 1 , in which a single target item appeared against a background of homogeneous nontargets. Therefore, as in Experiment 2, the targets in this design were not themselves singletons, because they were not unique on the target-defining dimension (frequency) and were not presented against a homogeneous background. Thus, as in
Experiment 2, singleton detection mode was discouraged in Experiment 3. However, in an important difference from Experiment 2, search difficulty was not increased in Experiment 3, because the search task remained the same as in Experiment 1, except for the addition of one target stimulus on some trials. Nevertheless, as in Experiment 2, if $\mathrm{AC}$ by the irrelevant intensity singleton depended on subjects adopting a singleton detection strategy, such capture should not have been found within the current design.

In addition, as in Bacon and Egeth's (1994) Experiment 2 , we included some search arrays containing only a single target (on $30 \%$ of the trials). These trials were identical to the trials used in Experiment 1, the only difference being that they were presented among other trials containing two targets, which should have prevented subjects from using a singleton detection strategy. If the singleton interference effect were to be eliminated in the subset of single-target trials within Experiment 3, this would reflect strategy differences, as the trials were otherwise identical to those of Experiment 1, in which a singleton interference effect was found.

\section{Method}

Participants. Twenty new participants took part in the experiment. Stimuli and Apparatus. The stimuli and apparatus used were the same as in Experiment 1.

Design and Procedure. The design was similar to that of Experiment 1. Each trial consisted of a sequence of five sounds, one after another. On $30 \%$ of trials a single low-frequency target appeared, as was the case throughout Experiment 1. This target appeared in the second, third, fourth, or fifth position with equal probability, and was just as likely to be long in duration as short. On the remaining $70 \%$ of trials, two identical low-frequency targets were present in the search array. These could also appear in the second, third, fourth, or fifth position with equal probability. On two-target trials, the targets were either both of long duration or both of short duration (with equal probability). Nontargets had a higher frequency than targets, with durations that were assigned as short or long at random. An irrelevant singleton, with the same frequency as the nontargets but with a higher intensity, was presented on $50 \%$ of trials. Singletons were of long or short duration with equal probability and were just as likely to occur before the targets as after them (and between them, in the case of two targets). The procedure was identical to that of Experiment 1, except that the experimental trials were run in eight blocks of 52 trials, for reasons of counterbalancing.

\section{Results and Discussion}

RTs. Table 2 shows mean RTs and error rates for Experiment 3 as a function of singleton presence (present vs. absent) and number of targets (one vs. two). A twoway within-subjects ANOVA was run on the RT data using these factors. ${ }^{4}$ As can be seen in Table 2, target responses were not any slower in the presence of a singleton $(M=318 \mathrm{msec})$ than in the absence of a singleton $(M=$ $319 \mathrm{msec}, F<1)$. Thus, as in Experiment 2, the singleton effect was eliminated where use of a singleton detection strategy was discouraged. There was a significant main effect of number of targets $\left[F(1,19)=44.25, M S_{\mathrm{e}}=525.48\right.$, $p<.05]$, such that responses were faster when there were two targets present in the sequence $(M=308 \mathrm{msec})$ than when there was only one target $(M=342 \mathrm{msec})$. This is probably because the target position is earlier on average 
Table 2

Averages of Subjects' Mean Reaction Times (RTs, in Milliseconds) With Standard Errors (SE) and Error Rates (\%E) for Experiment 3, As a Function of Singleton Presence and Number of Targets

\begin{tabular}{|c|c|c|c|c|c|c|}
\hline \multirow{3}{*}{$\begin{array}{c}\text { Number of } \\
\text { Targets }\end{array}$} & \multicolumn{6}{|c|}{ Singleton Condition } \\
\hline & \multicolumn{3}{|c|}{ Absent } & \multicolumn{3}{|c|}{ Present } \\
\hline & RT & $S E$ & $\% \mathrm{E}$ & RT & $S E$ & $\% \mathrm{E}$ \\
\hline 1 & 344 & 27 & 6 & 341 & 28 & 7 \\
\hline 2 & 309 & 24 & 4 & 308 & 25 & 5 \\
\hline
\end{tabular}

in two-target trials than in one-target trials, and participants might therefore begin to prepare responses earlier on two-target trials. There was no interaction between the two factors $(F<1)$.

Experiment 3 included trials in which there was only one target present. These trials are directly comparable with those of Experiment 1, in which there was always only one target present. Therefore, any difference in performance between the two conditions would strongly suggest an effect of strategy. A one-way within-subjects ANOVA, using the one-target RT data from Experiment 3, found no significant effect of singleton presence $(F<1$; see Table 2), indicating that even on trials that are directly comparable with Experiment 1, the singleton effect was not replicated.

A mixed-model ANOVA comparison of the effects of singleton presence between Experiment 1 and Experiment 3 found a significant interaction $[F(1,30)=4.36$, $\left.M S_{\mathrm{e}}=1,535.00, p<.05\right]$. This confirmed that the singleton effect in Experiment 1, in which participants were able to use a singleton detection strategy, was significantly reduced in Experiment 3, where this strategy was made unavailable. This result is in line with the findings of Experiment 2 , in which singleton detection strategy was also prevented and the singleton interference effect was significantly reduced. However, in contrast to the betweenexperiments analysis of Experiments 1 and 2, the present analysis revealed no main effect of experiment $(F<1)$, indicating that performance was comparable between Experiments $1(M=310 \mathrm{msec})$ and $3(M=319 \mathrm{msec})$. Moreover, RTs from one-target trials with the target in position 3 or $4(M=331 \mathrm{msec})$ were not significantly different from trials in Experiment $1[M=310 \mathrm{msec}$, $t(30)<1]$, with which they are directly comparable.

Errors. Overall error rates in the present experiment were comparable with those of Experiment 1 ( $M$ for both $=5 \%$ ), and, as in that experiment, a two-way withinsubjects ANOVA, with the factors of singleton presence (present vs. absent) and number of targets (one vs. two), revealed no effect of singleton presence $[F(1,19)=2.99$, $\left.M S_{\mathrm{e}}=0.001, p=.10\right]$ (see Table 2$)$. There was a significant main effect of number of targets $[F(1,19)=13.32$, $\left.M S_{\mathrm{e}}=0.001, p<.05\right]$, with higher error rates on onetarget trials $(M=7 \%)$ than on two-target trials $(M=$ $4 \%$ ). This is likely because of an increased probability of correct target identification, due to the redundant target presentation on two-target trials. Importantly, as with the RTs, there was no interaction between the number of tar- gets and singleton presence $(F<1)$ : The singleton did not produce interference in either the one- or the two-target cases.

In line with the RTs, a one-way within-subjects ANOVA using the one-target error data from Experiment 3 found no significant effect of singleton presence $[F(1,19)=$ $\left.1.67, M S_{\mathrm{e}}=21.03, p>.20\right]$ (see Table 2). This reinforces the finding from the RT analysis that the singleton interference effect was not evident even on trials that were directly comparable to those used in Experiment 1 .

In conclusion, the results of the present experiment converge with those of Experiment 2 to demonstrate a clear reduction in RT singleton interference effects when subjects are forced out of singleton detection strategy, either by including more than one nontarget (Experiment 2) or by including more than one target (Experiment 3 ). The present experiment demonstrates this reduction in singleton interference, despite a level of task performance comparable to that of Experiment 1.

\section{EXPERIMENT 4}

Experiment 4 was designed to test whether the elimination of the singleton effect in Experiments 2 and 3 might have been due to a reduction in singleton salience, because the search sequences were more heterogeneous, rather than relating to a change in the available search strategy. Recall that Experiment 2 involved a greater number of stimulus tokens than Experiment 1, as it included nontargets and singletons of both high and low frequencies. Similarly, Experiment 3 involved two targets, again making the sequence more heterogeneous. Although the distractor singleton remained unique on the dimension of intensity, it is possible that it became less salient in a sequence that involved greater sound variation (even if this variation was on other dimensions) compared with a more homogenous sequence, as in Experiment 1. A reduction in singleton salience has been shown to reduce AC effects in visual search (e.g., Theeuwes, 1992) and it may therefore have been responsible for the reduction in singleton interference in the present experiments; see Theeuwes (2004) for a similar criticism of Bacon and Egeth's (1994) findings.

In order to examine this possibility, the following experiment included two types of block, one of which included twice as many stimulus tokens as the other. In the blocks that included twice as many stimulus tokens, the additional stimuli were defined on the task-irrelevant dimension of duration, so that not only did the singleton remain unique on the dimension of intensity, as had been the case in Experiments 2 and 3, but in addition the target remained unique on the dimension of frequency, constituting a change from Experiments 2 and 3. As the target remained unique, this manipulation permitted a singleton detection strategy while increasing the stimulus variation, hence potentially reducing the salience of the singleton distractor. Thus, if the elimination of singleton interference effects in Experiments 1 and 2 had been due to singleton detection strategy being discouraged in those experiments rather than to a reduction in singleton salience, single- 
ton interference should have been found in Experiment 4, even in blocks containing increased numbers of stimuli.

Note that, in order to ensure that the duration variation affected singleton salience without altering the target task, judgments about the dimension of duration had to be removed from the target task. In order to achieve this, we changed the task from Experiments 1-3 (in which participants had to respond according to whether the $480 \mathrm{~Hz}$ was of long or short duration) to a task in which participants simply had to detect the presence or absence of the $480-\mathrm{Hz}$ target. This leaves the dimension of duration "free" to be used as task-irrelevant variation.

We also changed the response collection in the present experiment, so that subjects were able to respond any time after target presentation. This allowed us to test the assumption that singleton interference effects observed here (Experiment 1) and in previous experiments (Dalton \& Lavie, 2004) are due to "online" AC rather than to interference with memory/retrieval processes. Any such memory effects are ruled out in the present experiment, because participants are able to respond as soon as the target appears.

\section{Method}

Participants. Eight new participants took part in the experiment. One was ruled out due to a mean RT $(885 \mathrm{msec})$ that was over 1.6 $S D$ s from the group mean RT $(M=660 \mathrm{msec}, S D=138 \mathrm{msec})$ and an error rate $(8 \%)$ that was $3 S D$ s from the group mean $(M=2 \%$, $S D=2 \%)$.

Stimuli and Design. As in Experiment 1, targets were defined by frequency, being lower $(480 \mathrm{~Hz})$ than nontargets $(780 \mathrm{~Hz})$. However, in a change from Experiment 2, targets were now always of long duration $(300 \mathrm{msec})$, as the task was to detect the presence of the target rather than to discriminate between two possible targets. On half of the experimental blocks, the nontargets all had durations of $300 \mathrm{msec}$. On the other half of blocks, nontarget durations were assigned at random as either short $(100 \mathrm{msec})$ or long $(300 \mathrm{msec})$, with the constraint that at least one short and one long nontarget appeared on every trial. Singletons were presented at the nontarget frequency $(780 \mathrm{~Hz})$ and duration $(100 \mathrm{msec}$ or $300 \mathrm{msec}$, depending on the block) but with higher intensity (approximately $85 \mathrm{~dB}$ SPL) than the other sounds (approximately $73 \mathrm{~dB}$ SPL). Sounds were separated by varying ISIs to preserve a constant SOA of $400 \mathrm{msec}$.

Targets appeared on $50 \%$ of trials, in either the third or fourth position with equal probability. A high-intensity singleton appeared on $50 \%$ of trials, either before or after the target with equal probability, and singleton presence and position were fully crossed with target presence and position. Participants searched each sequence for a target sound of lower frequency than the nontargets and were asked to decide as quickly and accurately as possible whether the target was present or absent. They responded by pressing 1 for target present or 2 for target absent, using the right index and middle finger respectively on the number keypad. In a change from previous experiments, responses on target-present trials were collected from target onset and participants were made aware that on these trials they could respond as soon as they had detected a target. Responses on target-absent trials were calculated from the onset of the last item in the sequence. (We assume that this item is equivalent to the target on target-present trials, as it must be processed before a response can be made. Although in fact the target could never appear in the fifth serial position, participants were not made aware of this, and we therefore assume that they would have waited until the fifth item before responding). Six experimental blocks of 64 trials each were run, consisting of three blocks in which nontarget duration was varied and three in which nontarget duration remained constant. The experimental blocks were preceded by two practice blocks, each containing 16 trials. Order of presentation of blocks was counterbalanced across participants.

\section{Results and Discussion}

RTs. Table 3 shows mean RTs and error rates for Experiment 4 as a function of singleton presence, target presence, and nontarget duration (varied vs. constant). A threeway within-subjects ANOVA was run on the RT data using these factors. ${ }^{5}$ This revealed a significant main effect of singleton presence $\left[F(1,6)=6.23, M S_{\mathrm{e}}=345.20, p<\right.$ $.05]$, indicating that responses were slower when the singleton was present than when it was absent (see Table 3). Note that, in replicating our previous findings of singleton interference using a design in which subjects could respond as soon as the target was presented, the present experiment casts doubt on the suggestion that previous effects could be explained in terms of memory/retrieval processes.

There was also an overall main effect of nontarget duration $\left[F(1,6)=5.97, M S_{\mathrm{e}}=5,077.98, p=.05\right]$, such that responses were slower when the nontargets varied in duration $(M=683 \mathrm{msec})$ than when the nontargets were of constant duration $(M=637 \mathrm{msec})$. However, singleton presence did not interact with nontarget duration $\left[F(1,6)=2.94, M S_{\mathrm{e}}=120.81, p=.14\right]$, suggesting that the singleton interference effect was unaffected by whether nontarget duration was varied ( $M$ interference effect $=18 \mathrm{msec})$ or constant $(M$ interference effect $=$ $8 \mathrm{msec}$ ). An account of the results of Experiment 2 in terms of reduced singleton salience associated with increased variation in the nontargets would predict a reduced interference effect in blocks where the nontarget duration was varied in comparison with blocks where the nontarget duration was held constant. The present finding of comparable effects in both types of block therefore rules out this account.

There was no effect of target presence $(F<1)$, despite a numerical trend for slower responses when the target was absent $(M=689 \mathrm{msec})$ than when it was present $(M=631 \mathrm{msec})$. A closer inspection of the individual data suggested that the trend did not reach significance because two participants showed large effects in the opposite direction, with faster responses on target-absent

Table 3

Averages of Subjects' Mean Reaction Times (RTs, in Milliseconds), With Standard Errors ( $S E)$ and Error Rates (\%E) for Experiment 4, As a Function of Nontarget Duration (Varied or Constant), Singleton Presence, and Target Presence

\begin{tabular}{ccccccccc}
\hline & & \multicolumn{4}{c}{ Singleton Condition } \\
\cline { 3 - 6 } \cline { 3 - 6 } Nontarget & & \multicolumn{4}{c}{ Absent } & & \multicolumn{3}{c}{ Present } \\
\cline { 3 - 7 } Duration & Target & RT & $S E$ & $\% \mathrm{E}$ & & RT & $S E$ & $\% \mathrm{E}$ \\
\hline Varied & Absent & 719 & 68 & 1 & & 711 & 65 & 2 \\
& Present & 630 & 67 & 1 & & 672 & 70 & 1 \\
Constant & Absent & 668 & 51 & 2 & & 656 & 50 & 2 \\
& Present & 598 & 67 & 2 & & 624 & 74 & 2 \\
Average & & 654 & 51 & 2 & & 666 & 54 & 2 \\
\hline
\end{tabular}


(vs. -present) trials. It is possible that these participants picked up on the fact that the target never appeared in the fifth serial position and that they were therefore able to begin responding on presentation of the fourth item in the sequence. Our method of measuring RTs on target-absent trials from the fifth item in the stream would therefore underestimate RTs in this case. Target presence interacted with singleton presence $\left[F(1,6)=10.53, M S_{\mathrm{e}}=645.73\right.$, $p<.05]$, such that there was no significant difference between singleton-absent $(M=694 \mathrm{msec})$ and singletonpresent trials $(M=684 \mathrm{msec})$ when the target was absent $[t(6)=1.59, p>.15]$, whereas there was a large difference between singleton-absent $(M=614 \mathrm{msec})$ and singleton-present trials $(M=648 \mathrm{msec})$ when the target was present. The finding that the singleton effect was confined to target-present trials appears to be an anomalous result, as in our previous experiments using auditory detection tasks singleton effects tended to be stronger when the target was absent rather than present (e.g., Dalton \& Lavie, 2004, Experiments 1A, 1B, and 2B). The apparently anomalous result from the present experiment might be related to the change in response collection, whereby participants were now able to respond as soon as the target had been presented (in contrast to previous experiments, in which responses were only accepted at the end of the sequence). It is possible, for example, that this change may have increased the sensitivity of the target-present trials, leaving them more open to singleton interference than target-present trials in previous experiments. No other main effects or interactions were significant ( $p>.20$ for all comparisons).

Errors. Error rates were very small in the present experiment and a three-way within-subjects ANOVA with the factors of singleton presence (present vs. absent), target presence (present vs. absent) and nontarget duration (varied vs. constant) found no significant effects or interactions ( $p>.20$ for all comparisons).

In conclusion, Experiment 4 shows significant RT interference associated with the presence of irrelevant intensity singletons in an auditory frequency detection task. This finding reinforces the results of Experiment 1, in suggesting that auditory feature singletons capture attention as long as the target of the search is also itself a feature singleton. These findings are particularly interesting in confirming that the singleton effect persists when subjects are able to respond immediately after target presentation, rather than having to wait until the end of the sequence. This strengthens our claim that the effects reported here relate to "online" attentional effects rather than to interference in memory or retrieval processes.

However, the critical finding from the present experiment was that the singleton interference persisted in blocks of trials where the nontargets varied in duration, providing twice as many types of nontarget as in blocks where the nontargets were held constant. If the elimination of a singleton effect in Experiment 2 was due to a reduction in salience of the singleton, because of an increased number of nontarget stimuli, a similar effect should have been found in the present experiment. However, by contrast, this experiment showed no reduction of singleton effect associated with the addition of extra nontarget stimuli, suggesting that the elimination of the singleton effect in Experiments 2 and 3 was specifically due to the presence of heterogeneity on the target dimension (discouraging a singleton detection strategy), rather than to a general effect of greater heterogeneity of the sounds presented.

\section{GENERAL DISCUSSION}

Experiment 1 presented results from an auditory search task that differed from Dalton \& Lavie's (2004) original task in that the dimension used for target selection (frequency) was differentiated from that used for target responses (duration). This separation of target- and response-defining dimensions was necessary to allow us to manipulate search strategy in subsequent experiments without changing the response demands of the task. Experiment 1 found significant interference effects due to the presence of an irrelevant intensity singleton in this modified auditory search task, replicating previous demonstrations of auditory AC (Dalton \& Lavie, 2004). By contrast, Experiment 2 found no singleton effect in a task that was identical to that of Experiment 1, except for the inclusion of low-frequency nontargets in addition to highfrequency nontargets. Experiment 3 also eliminated the singleton interference effect by including trials in which two targets appeared.

The elimination in Experiments 2 and 3 of the singleton effect found in Experiment 1 is most likely to be due to the different strategies available in the two experiments. Participants in Experiment 1 could have adopted a singleton detection strategy, because the targets themselves were reliable feature singletons. However, because in Experiments 2 and 3 the targets were no longer unique feature singletons, the use of a singleton detection strategy would have been discouraged, leaving subjects less open to distraction by irrelevant singletons.

Although the reduction of the singleton effect in Experiment 2, by comparison with Experiment 1, may have been due in part to increased task difficulty and/or reduced singleton salience (due to the complex sequences used in Experiment 2), the convergence of results across Experiments 2, 3, and 4 makes it unlikely that the reduction in singleton effects seen in all three experiments can be explained solely in these terms. This is because Experiment 3 replicated the reduction in singleton interference without an associated increase in task difficulty, and Experiment 4 found that increased sequence complexity, achieved by increasing the number of stimulus tokens through introduction of variation on a nontarget dimension, did not affect the size of the singleton effect.

Further support for an explanation in terms of search strategy was provided from those trials in Experiment 3 in which only one target was present. The stimuli in these trials were very similar to trials in Experiment 1, with the only difference being that they were presented among two-target trials, whereas all trials in Experiment 1 had only single targets. As predicted by a search strategy explanation, the singleton distractor effect was eliminated in Experiment 3, even in this subset of one-target trials. 
The contrast between the presence of a singleton interference effect when single targets are used throughout the experiment (Experiment 1) and the elimination of this interference effect when the very same trials are presented in a block which includes a majority of two-target trials (Experiment 3) strongly suggests that the elimination of the singleton interference effect in Experiment 3 can be attributed to the search strategy adopted throughout that experiment.

It is important to note that the irrelevant singletons used here were of higher intensity than the rest of the sounds. It seems likely from an intuitive perspective that highintensity sounds might be particularly salient and therefore might be particularly good at capturing attention. Nevertheless, even capture by high-intensity sounds can be eliminated simply by a change in search strategy.

The present auditory findings allow for interesting comparison with results from the visual domain. Since hearing is free from the spatial restrictions of the other senses, it can monitor in all directions, whereas vision - the only other sense that can operate at long range - is restricted to a much smaller area of space. As such, it is likely that an important function of hearing is to provide organisms with advance notice of sudden changes in the environment. We might therefore expect auditory attention to be particularly open to $\mathrm{AC}$ by singleton sounds and thus perhaps less open to top-down influences. However, in contrast to this expectation, the present results demonstrate that subjects can avoid auditory AC by high-intensity singletons when they are encouraged to select a search strategy that does not emphasise singleton detection. The results therefore suggest that both auditory and visual AC processes are open to influence by the top-down factor of search strategy rather than being purely stimulus driven.

In demonstrating similarities between $\mathrm{AC}$ processes in vision and hearing, the present findings fit well with a recent study by Watkins, Dalton, Lavie, and Rees (2006), demonstrating that auditory AC by irrelevant singletons leads to activation of a dorsal fronto-parietal network similar to that activated during visual capture (e.g., de Fockert, Rees, Frith, \& Lavie, 2004). The findings also add more generally to a growing body of auditory research demonstrating auditory attentional phenomena that are analogous to well-researched visual phenomena. For example, researchers have found auditory analogues for the attentional blink (e.g., Arnell \& Larson, 2002; Duncan et al., 1997; Soto-Faraco \& Spence, 2002), change blindness (e.g., Vitevitch, 2003), AC by uninformative spatial cues (e.g., Spence \& Driver, 1997), and differences between search patterns for feature targets and for conjunction targets (e.g., Dyson \& Quinlan, 2003). It is perhaps unsurprising that attentional processes such as feature integration, change blindness, and attentional blink progress along similar lines in both vision and hearing, as these processes operate at a level that is likely to be largely independent of early sensory processing. In confirming that $\mathrm{AC}$ processes also operate similarly in vision and hearing, the present study provides further evidence that vision and hearing are subject to similar attentional constraints.

\section{AUTHOR NOTE}

We thank Chip Folk, Mari Riess Jones, and two anonymous reviewers for their helpful comments on an earlier draft of this work. This research was supported by an Engineering and Physical Sciences Research Council studentship (P.D.), an Economic and Social Research Council Postdoctoral Fellowship PTA-026-27-0550 (P.D.), and a Junior Research Fellowship from St. Anne's College, Oxford (P.D.). Address correspondence regarding this article to P. Dalton, Department of Psychology, Royal Holloway University of London, Egham, Surrey TW20 0EX, England (e-mail: polly.dalton@rhul.ac.uk).

\section{REFERENCES}

Arnell, K. M., \& Larson, J. M. (2002). Cross-modality attentional blinks without preparatory task-set switching. Psychonomic Bulletin \& Review, 9, 497-506.

BACON, W. F., \& EGETh, H. E. (1994). Overriding stimulus-driven attentional capture. Perception \& Psychophysics, 55, 485-496.

Bregman, A. S. (1990). Auditory scene analysis: The perceptual organization of sound. Cambridge, MA: MIT Press.

Broadbent, D. E., \& Broadbent, M. H. P. (1987). From detection to identification: Response to multiple targets in rapid serial visual presentation. Perception \& Psychophysics, 42, 105-113.

Chun, M. M. (1997). Temporal binding errors are redistributed by the attentional blink. Perception \& Psychophysics, 59, 1191-1199.

Cusack, R., \& CARLYon, R. P. (2003). Perceptual asymmetries in audition. Journal of Experimental Psychology: Human Perception \& Performance, 29, 713-725.

Dalton, P., \& LaVie, N. (2004). Auditory attentional capture: Effects of singleton distractor sounds. Journal of Experimental Psychology: Human Perception \& Performance, 30, 180-193.

Dalton, P., \& Lavie, N. (2006). Temporal attentional capture: Effects of irrelevant singletons on rapid serial visual search. Psychonomic Bulletin \& Review, 13, 881-885.

de Fockert, J., Rees, G., Frith, C., \& Lavie, N. (2004). Neural correlates of attentional capture in visual search. Journal of Cognitive Neuroscience, 16, 751-759.

DunCAN, J., MARTENS, S., \& WARD, R. (1997). Restricted attentional capacity within but not between sensory modalities. Nature, 387, 808-810.

Dyson, B. J., \& Quinlan, P. T. (2003). Feature and conjunction processing in the auditory modality. Perception \& Psychophysics, 65 , 254-272.

Folk, C. L., Leber, A. B., \& Egeth, H. E. (2001). Capture at the fovea: Control settings and the attentional blink. Paper presented at the 42nd Annual Meeting of the Psychonomic Society, Orlando.

Folk, C. L., Leber, A. B., \& Egeth, H. E. (2002). Made you blink! Contingent attentional capture produces a spatial blink. Perception \& Psychophysics, 64, 741-753.

Folk, C. L., \& Remington, R. W. (1998). Selectivity in distraction by irrelevant featural singletons: Evidence for two forms of attentional capture. Journal of Experimental Psychology: Human Perception \& Performance, 24, 847-858.

Folk, C. L., Remington, R. W., \& Johnston, J. C. (1992). Involuntary covert orienting is contingent on attentional control settings. Journal of Experimental Psychology: Human Perception \& Performance, 18, 1030-1044.

Folk, C. L., Remington, R. W., \& Wright, J. H. (1994). The structure of attentional control: Contingent attentional capture by apparent motion, abrupt onset, and color. Journal of Experimental Psychology: Human Perception \& Performance, 20, 317-329.

Ghorashi, S. M. S., Zuvic, S. M., Visser, T. A. W., \& Di Lollo, V. (2003). Focal distraction: Spatial shifts of attentional focus are not required for contingent capture. Journal of Experimental Psychology: Human Perception \& Performance, 29, 78-91.

Joliceur, P. (1998). Modulation of the attentional blink by on-line response selection: Evidence from speeded and unspeeded Task deci- $_{1}$ sions. Memory \& Cognition, 26, 1014-1032.

Kubovy, M. (1981). Concurrent-pitch segregation and the theory of indispensable attributes. In M. Kubovy \& J. R. Pomerantz (Eds.), Perceptual organization (pp. 55-96). Hillsdale, NJ: Erlbaum.

LAMING, D. R. J. (1968). Information theory of choice-reaction times. London: Academic Press. 
Maki, W. S., \& Mebane, M. W. (2006). Attentional capture triggers an attentional blink. Psychonomic Bulletin \& Review, 13, 125-131.

PAshler, H. (1988). Cross-dimensional interaction and texture segregation. Perception \& Psychophysics, 43, 307-318.

Raymond, J. E., Shapiro, K. L., \& Arnell, K. M. (1992). Temporary suppression of visual processing in an RSVP task: An attentional blink? Journal of Experimental Psychology: Human Perception \& Performance, 18, 849-860.

SCHARF, B. (1998). Auditory attention: The psychoacoustical approach. In H. Pashler (Ed.), Attention (pp. 75-117). Hove, U.K.: Psychology Press.

Soto-Faraco, S., \& Spence, C. (2002). Modality-specific auditory and visual temporal processing deficits. Quarterly Journal of Experimental Psychology, 55A, 23-40.

Spence, C., \& Driver, J. (1997). Audiovisual links in exogenous covert spatial orienting. Perception \& Psychophysics, 59, 1-22.

Theeuwes, J. (1991). Cross-dimensional perceptual selectivity. Perception \& Psychophysics, 50, 184-193.

Theeuwes, J. (1992). Perceptual selectivity for color and form. Perception \& Psychophysics, 51, 599-606.

TheEUwes, J. (2004). Top-down search strategies cannot override attentional capture. Psychonomic Bulletin \& Review, 11, 65-70.

VAN Noorden, L. P. A. S. (1975). Temporal coherence in the perception of tone sequences. Unpublished doctoral dissertation, Eindhoven University of Technology.

Vitevitch, M. S. (2003). Change deafness: The inability to detect changes between two voices. Journal of Experimental Psychology: Human Perception \& Performance, 29, 333-342.

Watkins, S., Dalton, P., Lavie, N., \& Rees, G. (2006). Brain mechanisms mediating auditory attentional capture in humans. Cerebral Cortex, e-pub ahead of print.

WeE, S., \& ChuA, F. K. (2004). Capturing attention when attention "blinks." Journal of Experimental Psychology: Human Perception \& Performance, 30, 598-612.

Welch, R. B., DuttonHurt, L. D., \& Warren, D. H. (1986). Contributions of audition and vision to temporal rate perception. Perception \& Psychophysics, 39, 294-300.

Woods, D. L., Alain, C., Diaz, R., Rhodes, D., \& Ogawa, K. H. (2001). Location and frequency cues in auditory selective attention. Journal of Experimental Psychology: Human Perception \& Performance, 27, 65-74.

\section{NOTES}

1. In the interests of consistency, all experiments in the present article use targets defined by frequency and singletons defined by intensity. Note, however, that auditory AC has previously been demonstrated using targets and singletons defined on several different feature dimensions (see Dalton \& Lavie, 2004).

2. It is unlikely that the tone sequences used here would have segregated into separate "streams" (e.g., Bregman, 1990) because the rate of presentation (with SOAs of $400 \mathrm{msec}$ ) is likely to have been too slow to support a strong streaming percept (e.g., van Noorden, 1975). Indeed, the majority of our experimental participants, as well as an independent auditory psychophysicist, reported perceiving the sequences used in the present experiment as integrated units (which untrained participants often referred to as "tunes").

3. A preliminary omnibus ANOVA was carried out on the RT data using all four possible experimental factors: singleton condition (absent vs. before-target vs. after-target); target position (3rd vs. 4th in the sequence); target duration (long vs. short); and singleton duration (long vs. short). The factors of target duration and singleton duration did not interact with the factor of theoretical interest (singleton condition) in any of the experiments; for the sake of simplicity, these factors are thus excluded from all the analyses. Although there was a significant interaction in Experiment 1 between target position and singleton condition, this interaction failed to replicate reliably across experiments (possibly because of the small number of trials per cell in the omnibus ANOVA) and we are therefore reluctant to draw any conclusions from it.

4. Because the majority of trials in Experiment 3 contained two targets, target position and singleton position (before vs. after the target) were ambiguous on a large number of trials. The removal of these ambiguous cases left too few trials to allow a reliable analysis of these factors.

5 . Because the singleton and target were only both present on $25 \%$ of trials, there were too few trials for a reliable analysis of singleton position (before vs. after the target) and target position.

(Manuscript received August 3, 2004; revision accepted for publication May 22, 2006.) 